\title{
Design, Fabrication, and Measurement of Two Silicon-Based Ultraviolet and Blue-Extended Photodiodes
}

\author{
Changping CHEN ${ }^{1,2,3}$, Han WANG ${ }^{1,2}$, Zhenyu JIANG ${ }^{1,2}$, \\ Xiangliang $\mathrm{JIN}^{1,2^{*}}$, and Jun $\mathrm{LUO}^{4}$ \\ ${ }^{1}$ Faculty of Materials, Optoelectronics and Physics, Xiangtan University, Xiangtan, 411105, China \\ ${ }^{2}$ Hunan Engineering Laboratory for Microelectronics, Optoelectronics and System on A Chip, Xiangtan, 411105, China \\ ${ }^{3}$ The forty-eighth Research Institute of China Electronics Technology Group Corporation, Changsha, 410111, China \\ ${ }^{4}$ Department of Precision Mechanical Engineering, Shanghai University, Shanghai, 200444, China \\ ${ }^{*}$ Corresponding author: Xiangliang JIN $\quad$ E-mail: jinxl@xtu.edu.cn
}

\begin{abstract}
Two silicon-based ultraviolet (UV) and blue-extended photodiodes are presented, which were fabricated for light detection in the ultraviolet/blue spectral range. Stripe-shaped and octagon-ring-shaped structures were designed to verify parameters of the UV-responsivity, UV-selectivity, breakdown voltage, and response time. The ultra-shallow lateral pn junction had been successfully realized in a standard $0.5-\mu \mathrm{m}$ complementary metal oxide semiconductor (CMOS) process to enlarge the pn junction area, enhance the absorption of UV light, and improve the responsivity and quantum efficiency. The test results illustrated that the stripe-shaped structure has the lower breakdown voltage, higher UV-responsicity, and higher UV-selectivity. But the octagon-ring-shaped structure has the lower dark current. The response time of both structures was almost the same.
\end{abstract}

Keywords: Ultraviolet/blue photodiode, ultraviolet responsivity, breakdown voltage, transient response, CMOS

Citation: Changping CHEN, Han WANG, Zhenyu JIANG, Xiangliang JIN, and Jun LUO, "Design, Fabrication, and Measurement of Two Silicon-Based Ultraviolet and Blue-Extended Photodiodes," Photonic Sensors, 2014, 4(4): 373-378.

\section{Introduction}

The ultraviolet detection technology is developed after the infrared and laser detection technologies, and ultraviolet (UV) detectors are hotly researched and used in many application areas, e.g., in environmental monitoring [1] or in biochemical analysis [2]. Photodiodes used for UV detecting are required to be sensitive to blue/UV radiation and blind to visible and near-infrared radiation. Different structures have been reported to realize UV silicon photodetectors. A. R. Pauchard [3] and Ayman Ghazi [4] proposed the silicon based UV photodiodes fabricated in the standard complementary metal oxide semiconductor (CMOS) process. The first ultraviolet avalanche photodiode in the CMOS technology was presented by A. Pauchard [5], and a responsivity of about $2.3 \mathrm{~A} / \mathrm{W}$ at $400 \mathrm{~nm}$ was achieved for a reverse bias of $19.1 \mathrm{~V}$. The development of phototransistors with the high responsivity for the $\mathrm{UV} / \mathrm{blue}$ spectral has attracted much interest in recent years [6, 7].

Several main and important problems should be considered in the study of the silicon-based UV photodiode in the standard CMOS process: (1) the noise and leakage current on the silicon surface $[8,9]$, (2) the spectral response range of the UV

Received: 23 April 2014 / Revised version: 6 June 2014

(C) The Author(s) 2014. This article is published with open access at Springerlink.com

DOI: $10.1007 / \mathrm{s} 13320-014-0199-7$

Article type: Regular 
photodetector and the penetration depth of UV light into the silicon material $[10,11],(3)$ the fabrication of the ultra-shallow pn junction in the CMOS process [12-14], and (4) a decrease in the breakdown voltage or operation voltage of the photodiode for silicon-on-a-chip ( $\mathrm{SoC}$ ) integration [15-17]. To optimize the noise performance of the UV photodiode, the processes of doping and isolation need to be improved. However, changing or adding process steps are too expensive. In this paper, an extremely thin layer with $\mathrm{p}^{+}$doping is injected on the crystal surface to reduce the defects, noise, and leakage current. The silicon-based UV photodiode can be fabricated with the readout circuit on a same chip when the working voltage is compatible to the CMOS process. Strip-shaped structures have been proposed to increase the absorption of UV light and reduce the breakdown voltage [3-5]. Various characteristics of the parameter were obtained for different UV photodiode structures.

In this paper, the stripe-shaped and octagon-ringshaped silicon-based UV and blue-extended photodiodes are presented, which were fabricated to verify and compare their parameters: UV-responsivity, UV-selectivity, breakdown voltage, and response time.

\section{Design and fabrication}

\subsection{Structure description}

Figure 1 shows the top view of the two proposed structures, Fig. 1(a) presents the stripe-shaped photodiode, and Fig. 1(b) presents the octagon-ring-shaped photodiode. They are actually conventional $\mathrm{p}^{+}-\mathrm{n}_{\text {well }}-\mathrm{p}_{\text {sub }}$ structures, and the schematic cross-section of them is shown in Fig. 1(c). The highly doped $\mathrm{p}^{+}$octagon-ring-shaped anodes are bounded by the $\mathrm{n}_{\text {well }}$ both laterally and vertically. The optical window of the structure is covered by an anti-reflection coating, which consists of a layer of $\mathrm{SiO}_{2}$ with a thickness of $60 \mathrm{~nm}$. The silicon surface is injected with high $\mathrm{p}^{+}$doping and forms an extremely thin layer. The width of each anode is $3.0 \mu \mathrm{m}$; the distance between two adjacent anodes is $1.5 \mu \mathrm{m}$. The total area of both photodiodes is about $\pi \cdot(50 \times 50) \mu \mathrm{m}^{2}$. The $\mathrm{p}_{\text {sub }}-\mathrm{n}_{\text {well }}$ junction of this device is short-circuited in order to achieve the better UV selectivity.

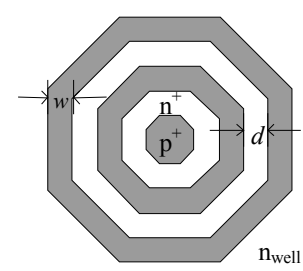

(a)

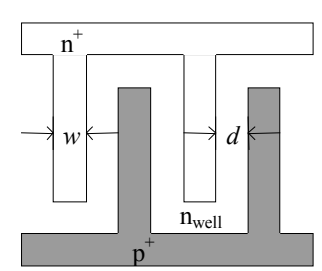

(b)

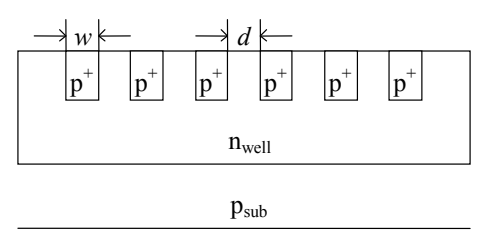

(c)

Fig. 1 Structures of the two photodiodes: (a) top view of the octagon-ring-shaped photodiode, (b) top view of the stripe-shaped photodiode, and (c) schematic cross-section of two UV photodiodes, where $w$ is the anode width and $d$ is the space between two anodes.

\subsection{Device fabrication}

The UV/blue-extended photodiodes were fabricated by the standard $0.5-\mu \mathrm{m}$ CMOS process. The microphotographs are shown in Fig. 2. Figure 2 (a) is the octagon-ring-shaped photodiode (Structure 1) with four laps of $\mathrm{p}^{+}$anodes, one $\mathrm{n}^{+}$ cathode, and one $\mathrm{p}^{+}$substrate, and Fig. 2(b) is the stripe-shaped photodiode (Structure 2), which has eight stripes of $\mathrm{p}^{+}$anodes, one stripe of $\mathrm{n}^{+}$cathode and one stripe of $\mathrm{p}^{+}$substrate. The two photodiodes have an equivalent area so as to make better comparisons. Their $\mathrm{p}^{+}$anodes are connected through metals, respectively. Their $\mathrm{n}^{+}$cathode and $\mathrm{p}^{+}$ substrate are short-circuited to make the photodiodes much more sensitive to UV light than to visible and near infrared radiation. Two pads are connected with the anode and cathode, respectively. The avalanche photodiode (APD) photodiode tester (GCAPD-B), oscilloscope (Tektronik MSO-2024), and micro- 
ammeter (HB-321) were used to test the proposed photodiodes.

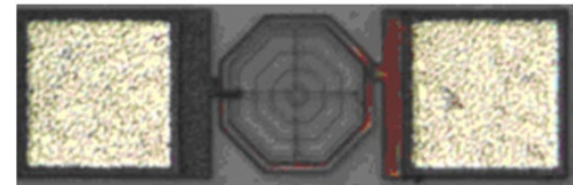

(a) Octagon-ring-shaped photodiode

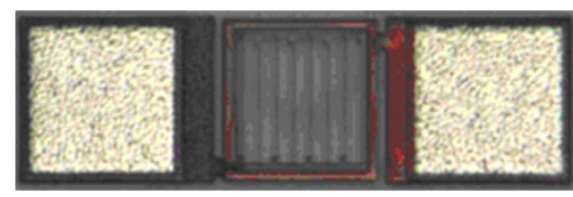

(b) Stripe-shaped photodiode

Fig. 2 Microphotographs of (a) the octagon-ring-shaped photodiode and (b) the stripe-shaped photodiode.

\section{Silicon test results}

\subsection{Spectral responsivity}

The spectral responsivities of those two UV/blue photodiodes are shown in Fig. 3. The reverse bias voltage was set at less than $16 \mathrm{~V}$, and the illumination intensity was set at $500 \mathrm{~lx}$. It can be seen that in the UV/blue spectrum $(0.3 \mu \mathrm{m}-0.5 \mu \mathrm{m})$, the spectral responsivity of Structure 2 was lager than that of Structure 1. The peak photocurrent responses of the two photodiodes were about $2.51 \times 10^{-8} \mathrm{~A}\left(\right.$ at $420 \mathrm{~nm}$ ) and $2.38 \times 10^{-8} \mathrm{~A}$ (at $450 \mathrm{~nm}$ ). However, the photocurrent response of Structure 2 becomes smaller than that of Structure 1 within the visible and IR range $(0.5 \mu \mathrm{m}-1 \mu \mathrm{m})$. At the wavelength of $1 \mu \mathrm{m}$, the photocurrent response of Structure 2 was $3.4 \times 10^{-11}$ A while Structure 1 got $5.41 \times 10^{-10} \mathrm{~A}$. In this paper, the UV-selectivity is defined as the ratio of the photocurrent response at $0.4 \mu \mathrm{m}$ and $1 \mu \mathrm{m}$. Structure 2 obtained an UV-selectivity of 560 while Structure 1 just obtained an UV-selectivity of 400 . To sum up, the stripe-shaped photodiode performs better in UV-reponsivity and UV-selectivity. Structure 1 has more areas of $\mathrm{p}^{+}$anode through calculation, which means that its dead layer [3] area is larger than that of Structure 2. The dead layer will decrease the UV-responsivity and UV-selectivity of the photodiode as described in [3].

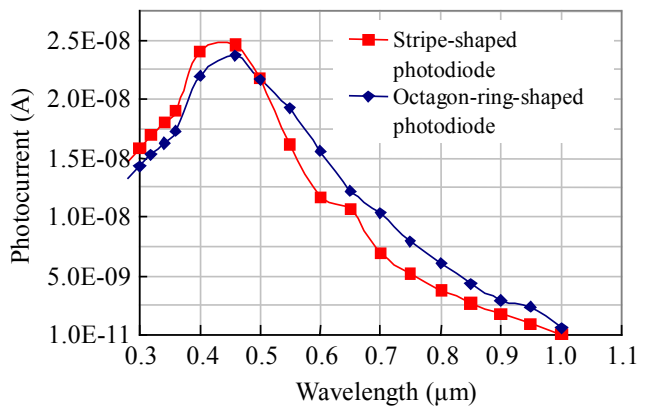

Fig. 3 Tested spectral responsivity (photocurrent response) of two UV/blue-extended photodiodes.

\subsection{I-V characteristic curves}

The comparison of the $I-V$ characteristics of those two UV photodiodes are shown in Fig. 4. It is evident to see that the breakdown voltage of Structure 1 is about $22.5 \mathrm{~V}$ while the other one of Structure 2 is about $17.2 \mathrm{~V}$. The photodiode with the stripe-shaped structure has the lower breakdown voltage than that of Structure 2. Because the rectangle mask was used to fabricate the stripe-shaped ships. The spherical pn junction was

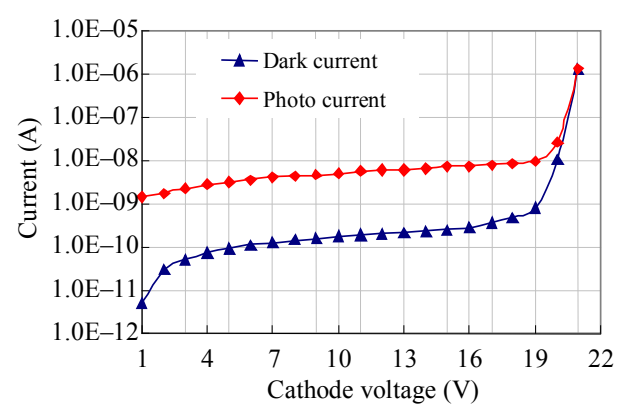

(a)

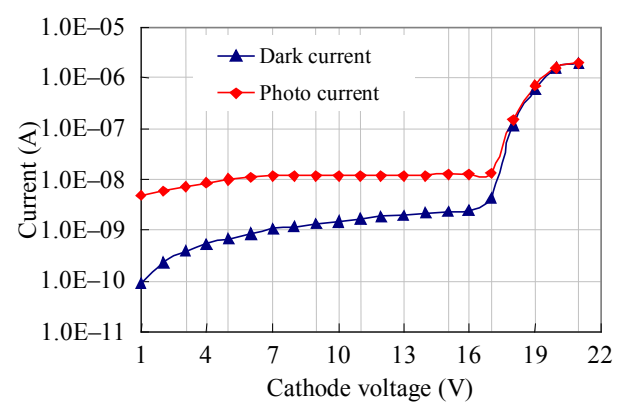

(b)

Fig. $4 I-V$ characteristics of the two photodiodes, (a) $I-V$ curves of Structure 1, breakdown voltage of which is about $20.5 \mathrm{~V}$, and (b) $I-V$ curves of Structure 2 whose breakdown voltage is about $17.2 \mathrm{~V}$. 
formed in angles of 90 degrees where the electric field was highly concentrated. It is known that the spherical pn junction has the largest electric field compared to the plane pn junction and cylindrical pn junction under a condition of the same substrate material, the same doping concentration, and the same bias voltage. Avalanche breakdown occurs easily in the spherical pn junction and cylindrical pn junction especially for very shallow pn junctions, which will reduce the avalanche voltage of the photodiode. However, edge breakdown will occur easily when the photodiode is under a voltage that is higher than the avalanche voltage, which increases the edge dark current and decreases the ratio of signal to noise. As a result, early edge breakdown should be avoided. Unfortunately, the stripe-shaped photodiode performs well but has a bigger dark current.

\subsection{Transient response}

The response time reflects how fast the UV photodiode is when it is biased and illuminated. Usually, the rise time and fall time are both considered. The response time of the proposed photodiodes are shown in Fig. 5. For Structure 1, its rise time and fall time are 8 nanoseconds and 2 nanoseconds, respectively. And for Structure 2, its rise time and fall time are 5 nanoseconds and 2 nanoseconds, respectively. As there are much more spherical pn junctions in Structure 2, the transport velocity of carriers are raised, and the response time is cut down. From another point of view, the octagon-ring-shaped photodiode has the highly symmetrical structure, the total time of diffusion and drifting are longer than that of the stripe-shaped photodiode, because there are much more phenomena of carriers diffusion in the octagonring-shaped photodiode. Although the difference of response time of both photodiodes is not clear, it can be predicted that the stripe-shaped photodiode would get even smaller response time if bigger structures or bigger arrays of the photodiode are fabricated.

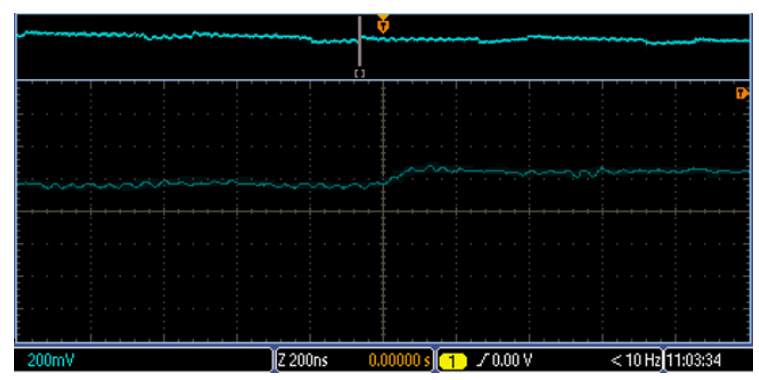

(a)

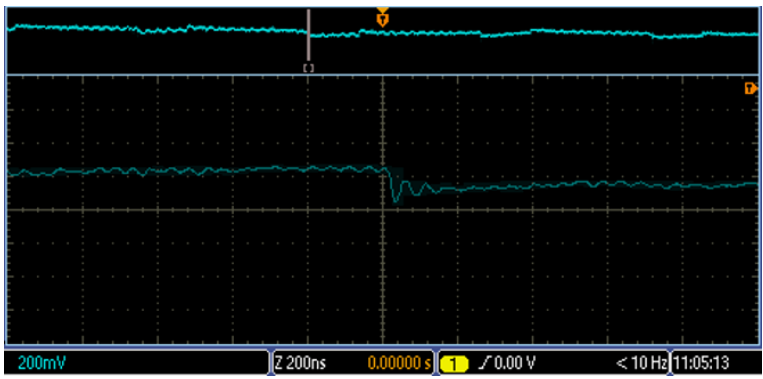

(b)

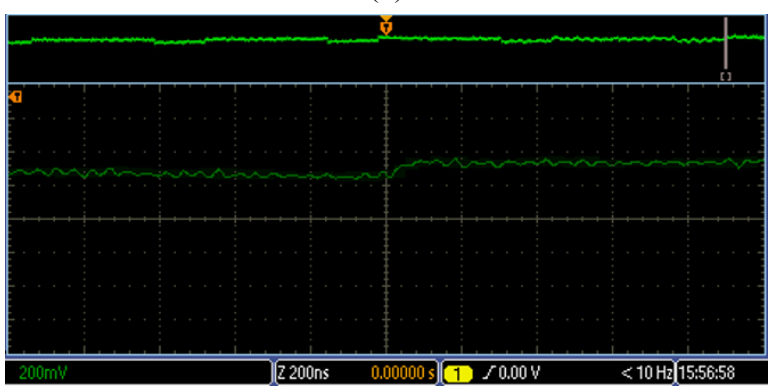

(c)

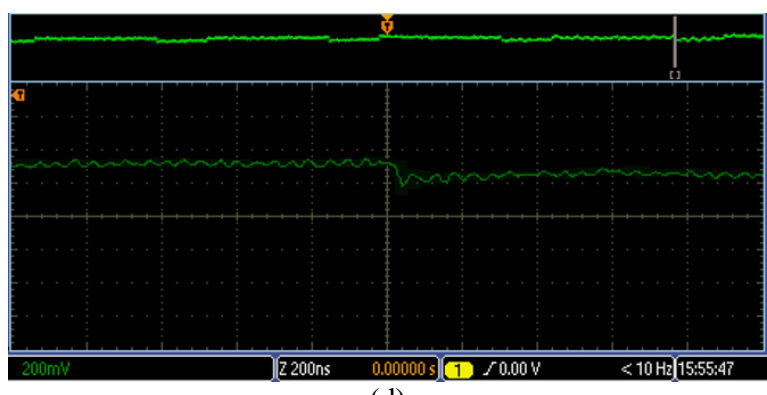

(d)

Fig. 5 Transient response of the two photodiodes: (a) rise time of Structure 1 is $8 \mathrm{~ns}$, (b) fall time of Structure 1 is $2 \mathrm{~ns}$, (c) rise time of Structure 2 is $5 \mathrm{~ns}$, and (d) fall time of Structure 2 is $2 \mathrm{~ns}$.

\section{Conclusions}

In conclusion, this paper presents and fabricated two silicon-based UV and blue-extended photodiodes. Stripe-shaped and octagon-ring-shaped structures were designed to verify and compare photodiode parameters of the UV-responsivity, 
UV-selectivity, breakdown voltage, and response time. The ultra-shallow lateral pn junction has been successfully realized in a standard $0.5-\mu \mathrm{m}$ CMOS process to enlarge the pn junction area, enhance the absorption of UV light, and improve the responsivity and quantum efficiency. The test results illustrate that the stripe-shaped structure has the lower breakdown voltage, higher UV-responsicity, and higher UV-selectivity. But the octagon-ringshaped structure has the lower dark current. The rise time of the stripe-shaped photodiode is small than that of the octagon-ring-shaped photodiode. Although there is not big difference in the response time of two structures, the conclusions in this paper can predict that the stripe-shaped photodiode would get even less response time if the bigger structures or bigger arrays of the photodiode are fabricated. The work in this paper is significant to help analyzing the performance of the silicon-based UV/blue photodiodes.

\section{Acknowledgment}

This work is supported by the State Key Program of National Natural Science of China (61233010), by the National Natural Science Foundation of China (61274043) and by the Program for New Century Excellent Talents in University of Ministry of Education of China (NCET-11-0975).

Open Access This article is distributed under the terms of the Creative Commons Attribution License which permits any use, distribution, and reproduction in any medium, provided the original author(s) and source are credited.

\section{References}

[1] E. Charbon, "Towards large scale CMOS single-photon detector arrays for lab-on-chip applications," Journal of Physics D: Applied Physics, 2008, 41(9): 1-9.

[2] Y. W. Chang, P. C. Yu, Y. T. Huang, and Y. S. Yang, "A CMOS-compatible optical biosensing system based on visible absorption spectroscopy," in IEEE
International Conference Electron Devices and Solid-State Circuits, Tainan, Dec. 20-22, pp. 1099-1102, 2007.

[3] A. Pauchard, P. A. Besse, and R. S. Popovic, "A silicon blue/UV selective stripe-shaped photodiode," Sens. Actuators, 1999, 76(1-3): 172-177.

[4] A. Ghazi, H. A. Zimmermann, and P. Seegebrecht, "CMOS photodiode with enhanced responsivity for the UV/blue spectral range," IEEE Transactions on Electron Devices, 2002, 49(7): 1124-1128.

[5] A. Pauchard, A. Rochas, Z. Randjelovic, P. A. Besse, and R. S. Popovic, "Ultraviolet avalanche photodiode in CMOS technology," in International Electron Devices Meeting 2000, San Francisco, USA, Dec. 10-13, pp. 709-712, 2000.

[6] Y. W. Chang and Y. T. Huang, "The ring-shaped CMOS-based phototransistor with high responsivity for the UV/blue spectral range," IEEE Photonics Technology Letters, 2009, 21(13): 899-901.

[7] G. Li, P. Feng, and N. Wu, "A novel monolithic ultraviolet image sensor based on a standard CMOS process," Journal of Semiconductors, 2011, 32(10): 105008-1-105008-6.

[8] T. Endoh, K. Hirose, and K. Shiraishi, "Physical origin of stress-induced leakage currents in ultra-thin silicon dioxide films," IEICE Transactions on Electronics, 2007, 90(5): 955-961.

[9] A. D. Foland, J. P. Alexander, P. I. Hopman, P. C. Kim, and C. W. Ward, "Radiation-induced surface leakage currents in silicon microstrip detectors," IEEE Transactions on Nuclear Science, 1996, 43(3): 1746-1750.

[10] G. E. Jellison, S. P. Withrow, J. W. McCamy, J. D. Budai, D. Lubben, and M. J. Godbole, "Optical functions of ion-implanted, laser-annealed heavily doped silicon," Physical Review B, 1995, 52(20): 14607.

[11] G. E. Jellison, "Optical functions of silicon determined by two-channel polarization modulation ellipsometry," Optical Materials, 1992, 1(1): 41-47.

[12] A. F. Vyatkin, V. I. Zinenko, A. N. Pustovit, and Y. A. Agafonov, "Ultra-shallow pn junction formation by ion implantation at high energy," in IEEE Proceedings of the 14th International Conference on Ion Implantation Technology, Taos, USA, Sep. 22-27, pp. 594-596, 2002.

[13] M. Hasumi, K. Ukawa, T. Sameshima, N. Sano, M. Naito, and N. Hamamoto, "Formation of shallow PN junction by cluster boron implantation and rapid annealing using infrared semiconductor laser," in InAIP Conference Proceedings, 2011, vol. 1321, pp. 109.

[14] K. Thorsten, A. Wei, and I. Ostermay. "Shallow PN junction formed by in situ doping during selective growth of an embedded semiconductor alloy by a 
cyclic growth/etch deposition process," U.S. Patent 8053273.8, 2011.

[15] I. Inoue, N. Tanaka, H. Yamashita, T. Yamaguchi, H. Ishiwata, and $\mathrm{H}$. Ihara, "Low-leakage-current and low-operating-voltage buried photodiode for a CMOS imager," IEEE Transactions on Electron Devices, 2003, 50(1): 43-47.

[16] J. Jung and A. Q. Huang, "Improved breakdownvoltage complementary MOSFET in a $0.18 \mu \mathrm{m}$ standard CMOS process for switch mode power supply (SMPS) applications," in Proceedings of Power Semiconductor Devices \& IC's, Jun. 14-18, pp. 239-242, 2009.

[17] F. P. Chou, G. Y. Chen, C. W. Wang, Z. Y. Li, Y. C. Liu, W. K. Huang, et al., "Design and analysis for a $850 \mathrm{~nm}$ Si photodiode using the body bias technique for low-voltage operation," Journal of Lightwave Technology, 2013, 31(6): 936-941. 